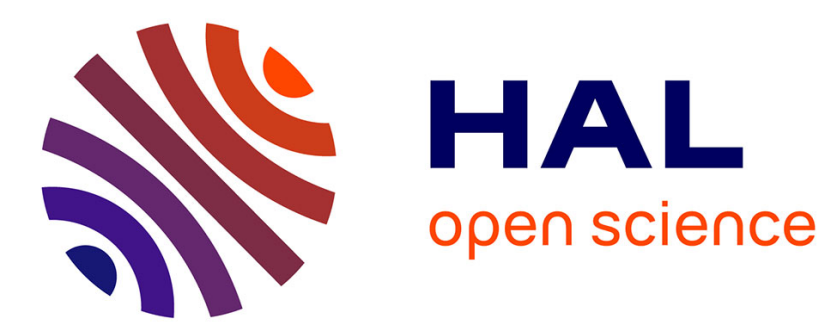

\title{
The lipid peroxidation products as possible markers of Alzheimer's disease in blood
}

\author{
A. Skoumalová, J. Ivica, P. Šantorová, E. Topinková, J. Wilhelm
}

\section{To cite this version:}

A. Skoumalová, J. Ivica, P. Šantorová, E. Topinková, J. Wilhelm. The lipid peroxidation products as possible markers of Alzheimer's disease in blood. Experimental Gerontology, 2010, 46 (1), pp.38. 10.1016/j.exger.2010.09.015 . hal-00647883

\section{HAL Id: hal-00647883 \\ https://hal.science/hal-00647883}

Submitted on 3 Dec 2011

HAL is a multi-disciplinary open access archive for the deposit and dissemination of scientific research documents, whether they are published or not. The documents may come from teaching and research institutions in France or abroad, or from public or private research centers.
L'archive ouverte pluridisciplinaire HAL, est destinée au dépôt et à la diffusion de documents scientifiques de niveau recherche, publiés ou non, émanant des établissements d'enseignement et de recherche français ou étrangers, des laboratoires publics ou privés. 


\section{Accepted Manuscript}

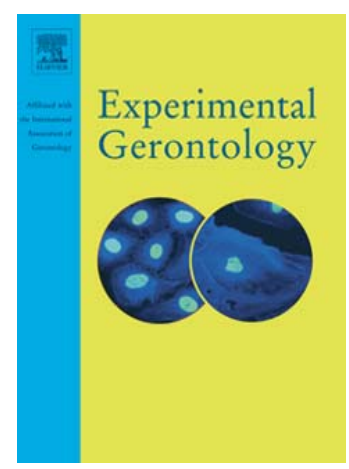

DOI:

S0531-5565(10)00346-3

The lipid peroxidation products as possible markers of Alzheimers disease in blood

A. Skoumalová, J. Ivica, P. Šantorová, E. Topinková, J. Wilhelm

doi: 10.1016/j.exger.2010.09.015

Reference: $\quad$ EXG 8814

To appear in: $\quad$ Experimental Gerontology

Received date: $\quad 8$ April 2010

Revised date: $\quad 16$ September 2010

Accepted date: 27 September 2010

Please cite this article as: Skoumalová, A., Ivica, J., Šantorová, P., Topinková, E., Wilhelm, J., The lipid peroxidation products as possible markers of Alzheimers disease in blood, Experimental Gerontology (2010), doi: 10.1016/j.exger.2010.09.015

This is a PDF file of an unedited manuscript that has been accepted for publication. As a service to our customers we are providing this early version of the manuscript. The manuscript will undergo copyediting, typesetting, and review of the resulting proof before it is published in its final form. Please note that during the production process errors may be discovered which could affect the content, and all legal disclaimers that apply to the journal pertain. 
The title of the paper: The lipid peroxidation products as possible markers of Alzheimer's disease in blood

Authors: A. Skoumalováa,", J. Ivica ${ }^{a}$, P. Šantorováa, E. Topinkováb, J. Wilhelmª

a Department of Medical Chemistry and Biochemistry, 2nd Faculty of Medicine Charles

Universtity in Prague, The Czech Republic

${ }^{b}$ Department of Geriatrics, 1st Faculty of Medicine Charles University and General Faculty Hospital in Prague, The Czech Republic

Address for mailing proofs: Dr. Alice Skoumalová, Department of Medical Chemistry and Biochemistry, Plzeňská 221/130, 15006 Prague, The Czech Republic

Telephone: +420257296306

Fax number: +420257210995

e-mail address: alice.skoumalova@1fmotol.cuni.cz

Keywords: Alzheimer's disease, diagnostic biomarkers, oxidative stress, lipofuscin-like pigments 


\begin{abstract}
:
Oxidative stress, which is present in Alzheimer's disease (AD), results in the formation of various end-products of free radical reactions with proteins and lipids. At present there are no reliable diagnostic biomarkers of $\mathrm{AD}$ in the blood. Therefore, specific products of lipid peroxidation in the blood of AD patients were investigated. Lipophilic extracts of erythrocytes in the group of patients with $\mathrm{AD}(\mathrm{n}=44)$ and age-matched controls $(\mathrm{n}=16)$ were studied. The end-products of lipid peroxidation, so called lipofuscin-like pigments (LFP), were analyzed by fluorescence spectroscopy. It was found that the level of these products is significantly increased in erythrocytes of $\mathrm{AD}$ patients compared to controls. LFP were furher separated by means of HPLC into individual fractions to study their composition in AD and controls. The specific fraction of LFP in AD patients, which was isolated, might represent a disease-specific product in the blood.
\end{abstract}




\section{Introduction:}

Alzheimer's disease (AD) represents the most common type of dementia and the total number of affected individuals increases worldwide due to demographic ageing. There is an increasing scientific evidence that novel antidementia drug therapies may slow down the progression of $\mathrm{AD}$ and some of them posess disease-modifying effect, particularly if treatment starts early in the course of disease. However, in most cases diagnosis is made only at later stages when significant cognitive impairment compromises patient's functioning (Shah et al., 2008), and neurodegeneration has progressed to an irreversible stage. It is hypothesized that treatment at early stages of AD may be more effective, and irreversible neuronal damage may be prevented. However, early and pre-clinical stages of AD are difficult to diagnose clinically and there are no reliable biomarkers for early AD diagnosis at present.

The assessment of $\mathrm{AD}$ biomarkers is complicated by diagnostic imprecision, the long asymptomatic prodromal stages, variability in clinical features and rates of progression, complex disease genetics and multiple molecular etiologies. On the other hand, oxidative stress that is present in AD (Praticò, 2008) results in the generation of oxidatively modified molecules in the early stages of the disease. In this context, the search for anomalous levels of free radical products facilitates the selection of biomarkers in AD. Several studies have shown that free radical products are present in the cerebrospinal fluid (CSF) of AD patients (Montine et al., 2002, Praticò et al., 1998, Strafacea et al., 2005). Nevertheless, CSF is not routinely collected in the evaluation of $\mathrm{AD}$, and lumbar puncture is not a widespread procedure. The identification of biomarker molecules in blood would be more widely applicable, and reduce the need for invasive, expensive or time-consuming testing.

Taking into account that the brain is rich in polyunsaturated fatty acids (PUFA), free radicals are expected to initiate lipid peroxidation. Occurence of lipid peroxidation in the 
brain in AD have been confirmed by several studies (for example Markesbery and Carney, 1999, Montine et al., 2002, Praticò and Delanty, 2000). In the course of these chain reactions toxic substances are formed which can further attack biomolecules and deepen the damage. Among these substances reactive aldehydes are found such as malondialdehyde (MDA) and 4-hydroxynonenal (HNE) that react with various cellular material to form stable end-products. These products have been originally called lipofuscin-like pigments (LFP) on the basis of similarity of their fluorescence spectra to lipofuscin (Chio and Tappel, 1969). Due to their native fluorescence they can be easily detected and have been used as an indicator of free radical damage to lipids in various tissues and pathologies (Goldstein and McDonagh, 1976, Skoumalova et al., 2003, Vasankari et al., 1995, Vesela et al., 2002, Wihlmark et al., 1996, Wilhelm and Herget, 1999).

Toxic aldehydes resulting from lipid peroxidation in the brain in AD can diffuse out from the primary site and attack erythrocytes in the blood stream. The presence of LFP in erythrocytes of dogs with canine counterpart of $\mathrm{AD}$ was previously found (Skoumalova et al., 2003). It implied the intention to extend this study to AD patients.

In the present study the LFP in erythrocytes of patients with AD were analysed by means of fluorescence spectroscopy. For a more detailed characterization of fluorescent properties of LFP, tridimensional spectral arrays and synchronous spectra were used. These methods revealed the complex nature of LFP and enabled detection of changes induced by oxidative stress in AD. Since LFP represent a mixture of compounds, it was separated by HPLC into individual fractions. The specific products found in erythrocytes in AD patients might be used for diagnostic purposes.

\section{Materials and methods}

\subsection{Patients}


Male and female subjects with established clinical diagnosis of "probable Alzheimer's disease" according to NINCDS-ADRDA criteria (McKhann et al. 1984), and with history of symptoms of AD for at least 6 months, and with Mini Mental State Examination (MMSE) (Folstein et al. 1975) score $\leq 24 / 30$ points $(\mathrm{N}=44)$ from out-patient memory clinic and inpatient of Department of Geriatric Medicine, General Faculty Hospital and First Faculty of Medicine of the Charles University in Prague were selected. Sociodemographic information and clinical characteristics of the AD (duration of symptoms, diagnosis, disease severity based on clinical symptoms and cognitive impairment using MMSE) were extracted from subject's medical record. Subjects with history and/or evidence of any other central nervous system disease that could be interpreted as the primary cause of dementia were not eligible including subjects with clinically significant focal neurological finding (with the exception of peripheral neuropathies) as well as subjects with possible, probable or definite vascular dementia according to NINCDS-AIREN criteria (Roman et al. 1993). Subjects with normal cognitive functions (MMSE $\geq 26 / 30$ points) and no subjective memory complains were used as control group $(\mathrm{N}=16)$. For both groups, patients with end-stage organ disease and advanced malignancies were not eligible.

For the study $5 \mathrm{ml}$ of blood samples were obtained, centrifuged, and sediment used for analysis. All samples were anonymously coded. Ethics Committee of VFN, Prague, approved the study.

\subsection{LFP fluorescence measuremets}

LFP in erythrocytes were determined according to the method of Goldstein and McDonagh (Goldstein and McDonagh, 1976). $0.5 \mathrm{ml}$ of frozen erythrocyte suspension was added to $6 \mathrm{ml}$ of chloroform-isopropanol mixture $(3: 2, \mathrm{v} / \mathrm{v})$ and extracted for $1 \mathrm{~h}$ on a motor- 
driven shaker. After extraction, $1 \mathrm{ml}$ of distilled water was added, mixed, and the mixture was centrifuged (400g; $10 \mathrm{~min}$ ). After centrifugation, the lower chloroform phase was separated and used for measurements.

Fluorescence excitation spectra of chloroform extracts were measured on the AMINCO-Bowman Series 2 spectrofluorometer coupled to a computer where the data were stored in the form of tridimensional spectral arrays. The excitation spectra were measured in the range of 250-400 $\mathrm{nm}$ for emission adjusted between 300-500 nm with a step of $10 \mathrm{~nm}$. The fluorometer was calibrated with $130 \mu \mathrm{M}$ quinine hemisulfate in $0.05 \mathrm{M} \mathrm{H}_{2} \mathrm{SO}_{4}$. Quantitative estimation of LFP was based on the excitation and emission maxima found in 3D spectral arrays. For determination of LFP concentration in erythrocytes the wavelenghts of the fluorescence maximum at 350/440 (excitation/ emission) were used. The ratio of fluorescence at $350 / 440 \mathrm{~nm}$ (fluorescence maximum) to $270 / 330 \mathrm{~nm}$ (fluorescence minimum) was used for quantification as an indicator of free radical damage.

Further analyses were performed by the measurement of synchronous fluorescence spectra. The fluorescence was measured with the constant difference of $25 \mathrm{~nm}$ between excitation and emission in the range of $250-500 \mathrm{~nm}$.

\subsection{HPLC analysis of LFP}

Erythrocyte chloroform extracts from patients with Alzheimer disease and agematched controls were evaporated under the stream of nitrogen. The evaporated sample was dissolved in approximately $2 \mathrm{ml}$ of running phase used in isocratic HPLC separation. For separation of LFP from erythrocytes a mixture of acetonitrile-methanol-water (50:10:40, v/v) was used. A Jasco (PU 2080, AS-2055) HPLC instrument equipped with fluorescence 
detector (FP-2020) set at 335/360 nm (excitation/emission) was used with a C18 column (4 x $250 \mathrm{~mm}$, Tessek, Czech Republic). Isocratic elution gave optimum separation at $0.2 \mathrm{ml} / \mathrm{min}$.

\subsection{Statistics}

Differences in measured parameters were analysed using the ANOVA with Bonferroni/Dunn post hoc test. The results are shown as means \pm SEM.

\section{Results}

Patients characteristics are described in Tab. 1. We investigated 60 subjects, including $44 \mathrm{AD}$ patients and 16 controls. None of the participants were current smokers and all of them were on the same diet. The group of AD patients includes 9 males and 35 females (mean age 83.95, SD 4.74, range 73-96). MMSE score was $16.11 \pm 5.75$. Some of the patients had diabetes $(n=15)$, dyslipidemia $(n=5)$ and/or hypertension $(n=30)$. The control group consisted of 5 males and 11 females. MMSE score was $28.88 \pm 1.73$. There were also subjects with diabetes $(n=2)$, dyslipidemia $(n=1)$ and/or hypertension $(n=6)$.

\subsection{Fluorescence spectra of LFP}

We analysed the fluorescent products of lipid peroxidation (LFP) as a marker of oxidative damage. The LFP in erythrocytes of AD patients and age-matched controls were studied by means of tridimensional fluorescence spectral arrays. Tridimensional fluorescence spectra reveal all the fluorophores present in a given sample and show the quantitative and qualitative changes resulting from oxidative damage. The spectral pattern of all individuals in a group was similar. Therefore, the characteristic examples of both groups are shown in Fig. 
1. Panel A is a characteristic spectrum of an AD patient, panel B shows a typical spectrum of age-matched control. In both cases, the excitation and emission maxima were found at 350 and $440 \mathrm{~nm}$, respectively. The fluorescence of the AD group was more pronounced than that of the control group. When the spectra of the control group are subtracted from that of the AD group, the result (panel C) shows the fluorescence produced as a consequence of AD. The fluorescence maximum is 350/440 (exitation/emission). This fluorescence corresponds to the group of fluorophores present in AD.

In order to further characterize LFP in erythrocytes of AD patients, their synchronous fluorescence spectra were measured. This method serves as a "fingerprint" of a given mixture and differences in these spectra indicate changes in the composition of the fluorophores. Fig. $\underline{2 \text { illustrates an example showing maximum difference of synchronous spectra of AD group }}$ (curve A) and the control group (curve B). Otherwise, there was great individual variability among the patients. It is apparent from Fig. 2 that greatest quantitative difference between the groups was found for the fluorophore $335 / 360 \mathrm{~nm}$ (excitation/emission).

\subsection{Quantitative analysis of LFP}

There were no statistically significant differences in LFP concentration within the experimental groups, e.g. the subgroup of $\mathrm{AD}$ patients with dyslipidemia did not differ from the rest of the group. Similar situation was observed in controls. Therefore the groups were evaluated without subdivisions.

For the quantitation of LFP concentration in controls and AD patients we used the fluorescence maxima found in tridimensional and synchronous spectral analysis at 350/440 $\mathrm{nm}$ and 335/360 nm (excitation/emission). The results are presented as a bar graph in Fig. 3. The concentration of the fluorophore F350/440 was significantly increased in AD patients (column A, $140 \%$ of controls, $\mathrm{P}<0,01$ ). Also the concentration of the second fluorophore 
F335/360, found in the synchronous spectra, is higher in the AD group (column A) compared to the control group $(113 \%, \mathrm{P}<0,05)$.

\subsection{HPLC analysis of LFP}

The LFP represents a mixture of many compounds. Spectrally characterized fluorophores can further be resolved by chromatographic methods such as HPLC. Both major fluorophores found in fluorescence spectra (F350/440 and F335/360) were analyzed. The greatest differences between $\mathrm{AD}$ group and controls during analysis of the fluorophore F335/360 were observed. Fig. 4 shows the most pronounced difference between tracings of AD group (curve A) and the control group (curve B). There was an intensive peak around 12 min in AD group (curve A) that was not observed in the controls (curve B). It was necessary to increase the sensitivity of the instrument about four-fold in order to show the details of the control tracing.

HPLC tracing of the fluorophore F 350/440 did not differ qualitatively between control and AD patients group. Thus, the major HPLC fraction of the fluorophore F335/360 found in $\mathrm{AD}$ patients might represent a disease-specific product with a potential of a marker of $\mathrm{AD}$.

\section{Discussion:}

As documented in a recent review (Gustaw-Rothenberg et al., 2010), oxidative stress and free radical damage is found early in the pathogenesis of $\mathrm{AD}$ and it is suggested that the products of oxidative damage might serve as a relevant group of biomarkers. Several studies have found signs of oxidative damage in AD patients. The aldehydic products of lipid 
peroxidation, especially HNE (McGrath et al., 2001), and MDA (Bourdel-Marchasson et al., 2001) were elevated in plasma. Concomitantly the antioxidants ascorbate and $\alpha$-tocopherol were decreased. The level of protein carbonyls and MDA in plasma was significantly higher in dementia compared to controls (Bermejo et al., 2008, Greilberger et al., 2008). The idea of general oxidative stress in $\mathrm{AD}$ was confirmed by detection of increased levels of isoprostanes in plasma and urine (Montine et al., 2002).

On the other hand, LFP represent a mixture of free radical products the composition of which is dependent on the kind of free radicals involved, and is tissue- or organ- specific (Wilhelm and Herget, 1999). Thus the methods able to resolve LFP into individual species might detect specific products related to AD pathogenesis. In our preceding study we found increased level of LFP in erythrocytes of dogs with canine counterpart of AD compared to age-matched control animals. Besides the quantitative difference the fluorescent species differed also in their fluorescence spectra, which further confirmed the disease specific product (Skoumalova et al., 2003).

Pathology can affect the production of fluorophores by two ways. The first possibility consists in the increase in quantity of a fluorophore present before the pathology has started. For example, there is a certain electron leak in mitochondria of a healthy subject that results in a certain basic level of a given fluorophore. If the pathology onset is accompanied with greater electron leak (increased lipid peroxidation), the composition of the fluorophore does not change, however, its concentration increases. This type of process could explain the quantitative increase in fluorophore $350 / 440 \mathrm{~nm}$ found in 3D spectra, and the fact that HPLC did not reveal a difference in its composition between controls and $\mathrm{AD}$ patients.

The second possibility of how pathology can influence a fluorophore formation consists in a new type of radical attack. For instance, new cellular compartment is being affected, thus new substrates are attacked, which results in the formation of a fluorophore not 
present in the healthy state. This type of process can explain the formation of fluorophore $335 / 360 \mathrm{~nm}$, as it was confirmed by HPLC only in AD patients.

Both types of fluorophores should be further studied as potential biomarkers of AD. The fluorophore $350 / 450 \mathrm{~nm}$ could be influenced by other non-specific pathologies related to free radical production. However, patients included in our study with cardiovascular disease, diabetes or dyslipidemia did not show increased levels of this fluorohore. As the increase in fluorophore $350 / 440 \mathrm{~nm}$ in $\mathrm{AD}$ patients is higher than that of fluorophore $335 / 360 \mathrm{~nm}$, the measurement of $350 / 440 \mathrm{~nm}$ offers higher sensitivity.

On the other hand, the fluorophore $335 / 360 \mathrm{~nm}$ appears to be specific to AD patients and this specificity should be tested on patients suffering from other types of neurodegenerative diseases in future studies.

The fluorescent products of lipid peroxidation represent important metabolites with potentially high diagnostic value. However, in order to realise their potential it will be necessary to characterize the chemical composition of these compounds. This has not yet been done for the compounds detected by means of fluorescence in complex biological preparations. Resolution of the lipophilic extracts from cells or tissues into individual LFP fractions by HPLC is a prerequisite for further characterization of these fractions by mass spectrometry and other analytical techniques. This will be the subject of future research in our laboratory. 
Acknowledgements:

This research was supported by The Czech Science Foundation, grant number 220032.

Disclosure Statement: There are no actual or potential conflicts of interests including any financial, personal or other relationships with other people or organizations within three years of beginning the work submitted that could inappropriately influence their work. Author's institutions do not have contracts relating to this research through which they or any other organization may stand to gain financially now or in the future. 
References:

Bermejo, P., Martín-Aragón, S., Benedí, J., Susín, C., Felici, E., Gil, P., Ribera, J.M., Villar, Á.M., 2008. Peripheral levels of Alzheimer's disease from Mild Cognitive Impairment. Free Radic. Res. 42, 162-170.

Bourdel-Marchasson, I., Delmas-Beauvieux, M-C., Peuchant, E., Richard-Harston, S., Decamps, A., Reignier, B., Emeriau, J-P., Rainfray, M., 2001. Antioxidant defences and oxidative stress markers in erythrocytes and plasma from normally nourished elderly Alzheimer patients. Age Ageing 30, 235-241.

Chio, K.S., Tappel, A.L., 1969. Synthesis and characterization of the fluorescent products derived from malonaldehyde and amino acids. Biochemistry 8, 2821-2826.

Folstein, M.F., Folstein, S.E., McHugh, P.R., 1975. "Mini-mental state”. A practical method for grading the cognitive state of patients for the clinician. J. Psychiatr. Res. 12 (3), 189-198. Goldstein, B.D., McDonagh, E.M., 1976. Spectrofluorescent detection of in vivo red cell lipid peroxidation in patients treated with diaminodiphenylsulfone. J. Clin. Invest. 57, 1302-1307. Greilberger, J., Koidl, C., Greilberger, M., Lamprecht, M., Schroecknadel, K., Leblhuber, F., Fuchs, D., Oettl, K., 2008. Malondialdehyde, carbonyl proteins and albumin-disulphide as useful oxidative markers in mild cognitive impairment and Alzheimer's disease. Free Radic. Res. 42, 633-638.

Gustaw-Rothenberg, K., Lerner, A., Bonda, D.J., Lee, H.G., Zhu, X., Perry, G., Smith, M.A., 2010. Biomarkers in Alzheimer's disease: past, present and future. Biomarkers Med. 4 (1), $15-26$.

Markesbery, W.R., Carney, J.M., 1999. Oxidative alterations inAlzheimer's disease. Brain Pahol. 9, 133-146. 
McGrath, L.T., McGleenon, B.M., Brennan, S., McColl, D., McIlroy, S., Passmore, A.P., 2001. Increased oxidative stress in Alzheimer's disease as assessed with 4-hydroxynonenal but not malondialdehyde. Q. J. Med. 94, 485-490.

McKhann G, Drachman D, Folstein M, Katzman R, Price D, Stadlan EM. Clinical diagnosis of Alzheimer's disease: report of the NINCDS-ADRDA work group under the auspices of Department of Health and Human Services Task Force on Alzheimer's Disease. 1984.

Neurology 34; 939-944.

Montine, T. J., Neely, M. D., Quinn, J. F., Beal, M. F., Markesbery, W. R., Roberts II, L. J., Morrow, J. D., 2002. Lipid peroxidation in aging brain and Alzheimer's disease. Free Radic. Biol. Med. 33, 620-626.

Praticò, D., Lee, V. M.-Y., Trojanowski, J. Q., Rokach, J., Fitzgerald, G. A., 1998. Increased F2-isoprostanes in Alzheimer's disease: evidence for enhanced lipid peroxidation in vivo. FASEB J. 12, 1777-1783.

Praticò, D., Delanty, N., 2000. Oxidative injury in Diseases of the central nervous system: focus on Alzheimer's disease. Am. J. Med. 109, 577-585.

Praticò, D., 2008. Evidence of oxidative stress in Alzheimer's disease brain and antioxidant therapy. Ann. N. Y. Acad. Sci. 1147, 70-78.

Roman et al. (1993) Vascular dementia: diagnostic criteria for research studies. Report of the National Institute of Neurological Communicative Disorders and Stroke-Association Internationale pour la Recherche' et l'Enseignement en Nourosciences (NINCDS-AIREN) International Workshop. Neurology, 43, 250-260.

Shah, R.S., Lee, H-G., Xiongwei, Z., Perry, G., Smith, M.A., Castellani, R.J., 2008. Current approaches in the treatment of Alzheimer's disease. Biomed. Pharmacother. 62, 199-207. 
Skoumalova, A., Rofina, J., Schwippelová, Z., Gruys, E., Wilhelm, J., 2003. The role of free radicals in canine counterpart of senile dementi of the Alzheimer type. Exp. Gerontol. 38, 711-719.

Strafacea, E., Matarresea, P., Gambardellaa, L., Vonaa, R., Sgadarib, A., Silverib, M. C., Malorni, W., 2005. Oxidative imbalance and cathepsin D changes as peripheral blood biomarkers of Alzheimer disease: A pilot study. FEBS Letters 579, 2759-2766.

Vasankari, T., Kujala, U., Heinonen, O., Kapanen, J., Ahotupa, M., 1995. Measurement of serum lipid peroxidation during exercise using three different methods: diene conjugation, thiobarbituric acid reactive material and fluorescent chromolipids. Clin. Chim. Acta 31, 6369.

Vesela, A., Wilhelm, J., Kawaciuk, I., 2002. Investigation of the role of free radical damage in patients after transurethral prostatectomy. J. Urol. 167, 221-222.

Wihlmark, U., Wrigstad, A., Roberg, K., Brunk, U.T., Nilsson, S.E., 1996. Formation of lipofuscin in cultured retinal pigment epithelial cells exposed to pre-oxidized photoreceptor outer segments. APMIS 104, 272-279.

Wilhelm, J., Herget, J., 1999. Hypoxia induces free radical damage to rat erythrocytes and spleen: analysis of the fluorescent end-products of lipid peroxidation. IJBCB 31, 671-681. 
Fig. 1 Fluorescence excitation spectral arrays of LFP in erythrocytes. Representative examples of the AD (panel A) and control (panel B) groups. Panel C shows the difference between spectrum A and B. The fluorescence maximum has been found at 350/440 nm (excitation/emission). Fluorescence intensity is given in arbitrary units.

Fig. 2 Synchronous fluorescence spectra of LFP in erythrocytes . Representative examples of the AD group (spectrum A) and control group (spectrum B). The fluorescence maximum has been found at $360 \mathrm{~nm}$ (emission) with the constant difference between excitation and emission of $25 \mathrm{~nm}$. Fluorescence intensity is given in arbitrary units.

Fig. 3 Quantitative expression of the LFP levels in erythrocytes. The quantitative analysis was done at two fluorescence maxima: 350/440 $\mathrm{nm}$ and 335/360 nm (excitation/emission).

Column A indicates the AD group, column B the control group. Statistical significance: * $\mathrm{P}<$ $0.05, * * \mathrm{P}<0.01$, related to controls.

Fig. 4 Chromatograms of erythrocyte chloroform extracts after HPLC separation with a fluorescence detection at 335/360 nm (excitation/emission). Panel A represents AD group, panel B shows control group.

Tab. 1 Characteristics of patients with Alzheimer's disease and controls 
Fig. 1

Skoumalová et al.
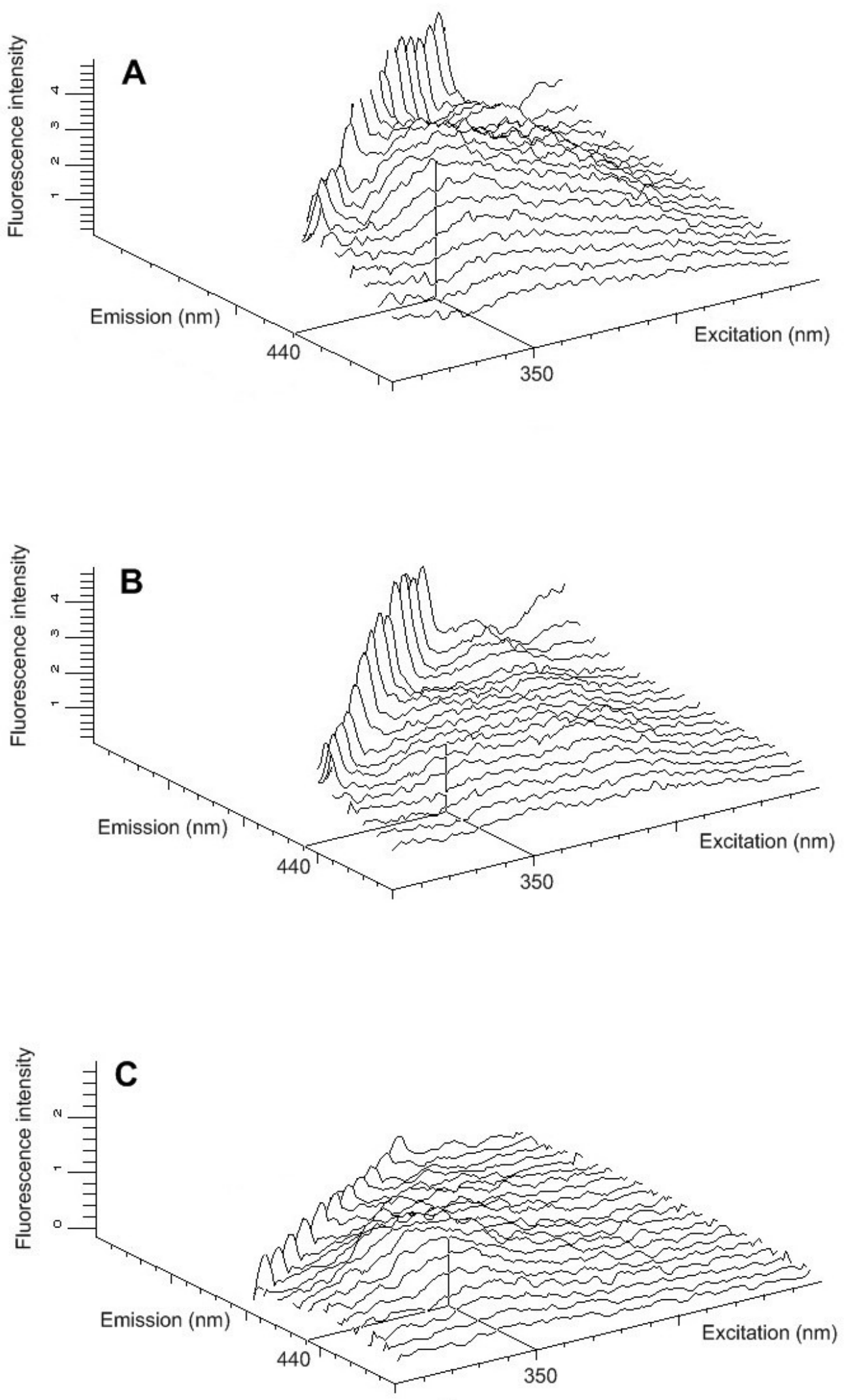
Fig. 2

Skoumalová et al.

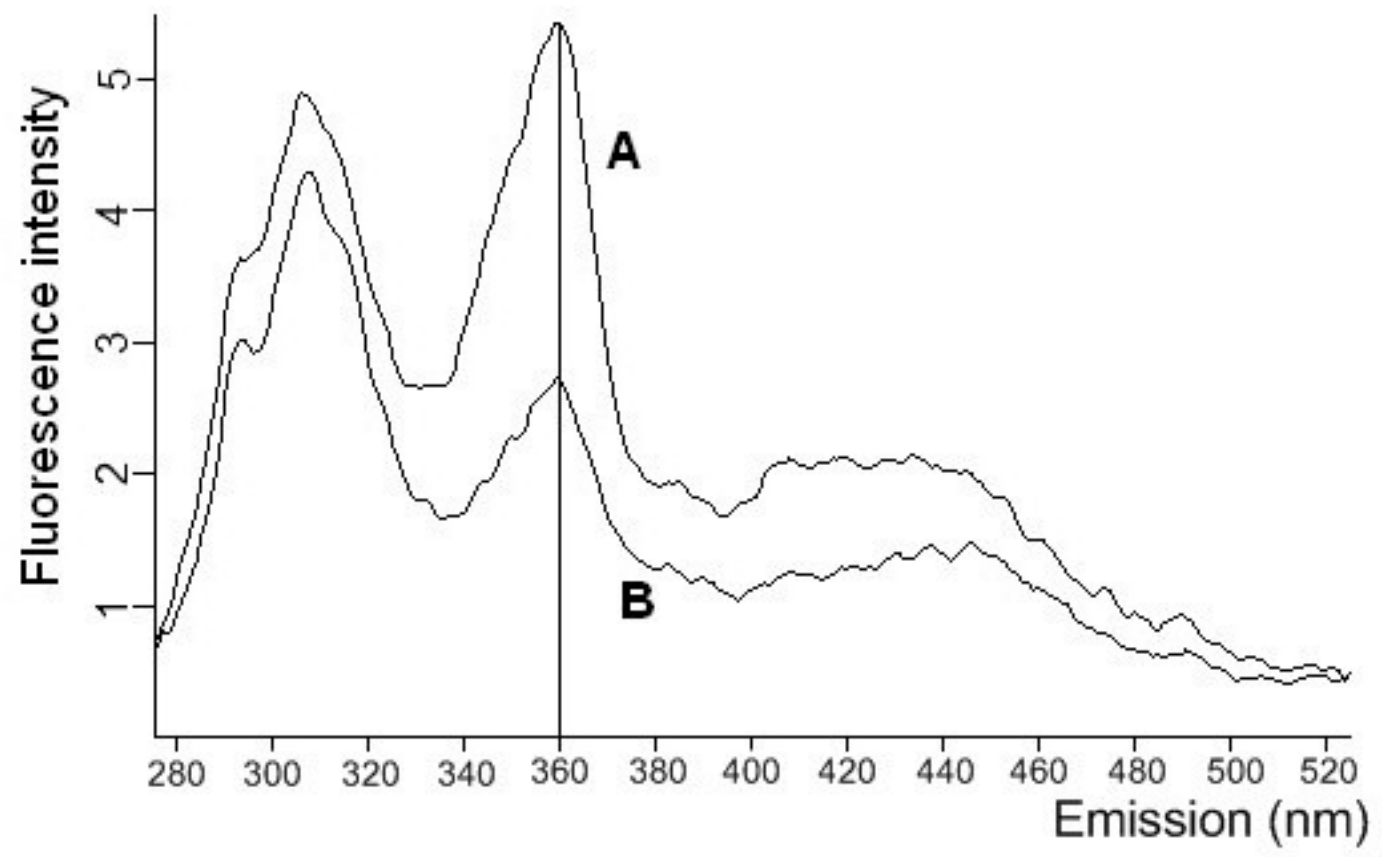


Fig. 3

Skoumalová et al.
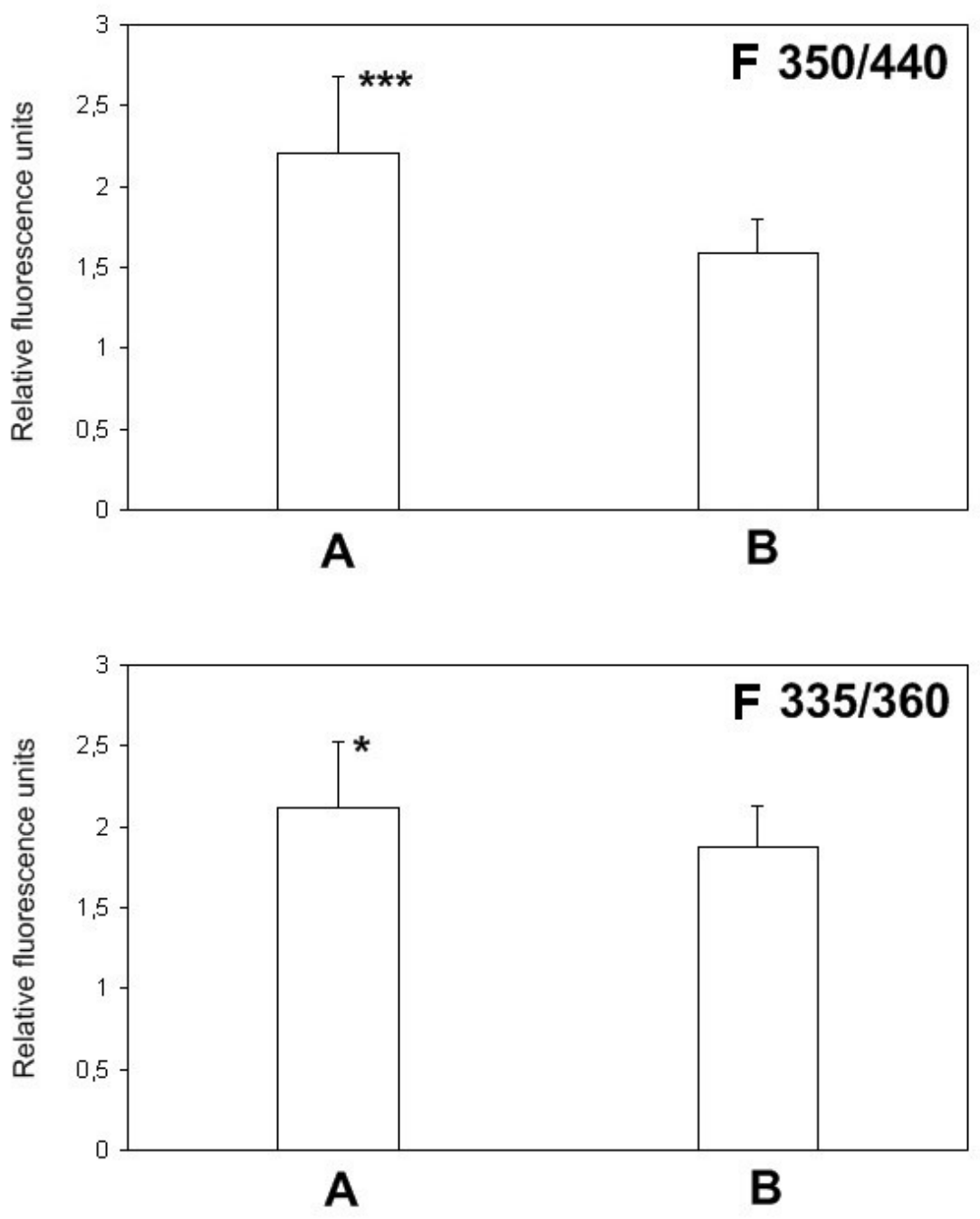


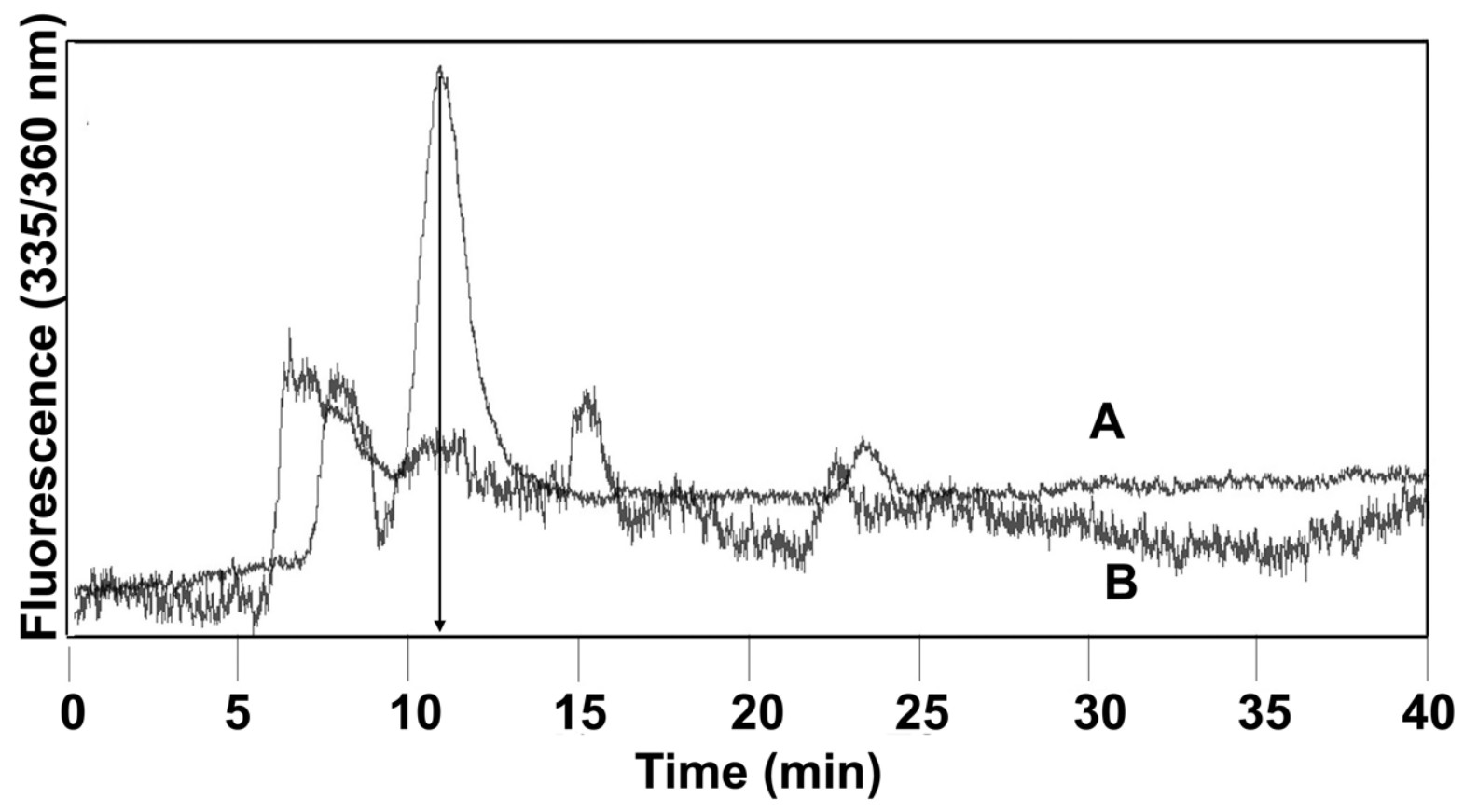

Fig. 4

\begin{tabular}{|l|c|c|}
\hline & Patients $(n=44)$ & Controls $(n=16)$ \\
\hline Mean age, years $( \pm$ SD) & $83.85 \pm 4.74$ & $76.44 \pm 9.53$ \\
\hline Sex ratio (men / women) & $9 / 35$ & $5 / 11$ \\
\hline MMSE $( \pm$ SD) & $16.11 \pm 5.75$ & $28.88 \pm 1.73$ \\
\hline Diabetes & $34 \%$ & $12.5 \%$ \\
\hline Dyslipidemia & $11 \%$ & $6.3 \%$ \\
\hline Hypertension & $68 \%$ & $37.5 \%$ \\
\hline
\end{tabular}

Table 1 


\section{Research Highlights}

- The level of end-products of lipid peroxidation is significantly increased in erythrocytes of Alzheimer's disease patients compared to controls

- The fluorophore 335/360 nm (excitation/emission) is specific for Alzheimer's disease and is separated into individual fractions

- The specific fraction of lipofuscin-like pigments in $\mathrm{AD}$ patients, which was isolated, might represent a disease-specific product in the blood 